\title{
Binomial Transforms of the Padovan and Perrin Matrix Sequences
}

\author{
Nazmiye Yilmaz and Necati Taskara \\ Department of Mathematics, Faculty of Science, Selcuk University, Campus, 42075 Konya, Turkey \\ Correspondence should be addressed to Nazmiye Yilmaz; nzyilmaz@selcuk.edu.tr
}

Received 18 July 2013; Accepted 13 September 2013

Academic Editor: Beong In Yun

Copyright (C) 2013 N. Yilmaz and N. Taskara. This is an open access article distributed under the Creative Commons Attribution License, which permits unrestricted use, distribution, and reproduction in any medium, provided the original work is properly cited.

We apply the binomial transforms to Padovan and Perrin matrix sequences. Also, the Binet formulas, summations, and generating functions of these transforms are found by recurrence relations. Finally, we illustrate the relations between these transforms by deriving new formulas.

\section{Introduction and Preliminaries}

There are so many studies in the literature that are concernes about the special number sequences such as Fibonacci, Lucas, Pell, Jacobsthal, Padovan, and Perrin (see, e.g., [1-4] and the references cited therein). In Fibonacci numbers, there clearly exists the term golden ratio which is defined as the ratio of two consecutive of Fibonacci numbers that converges to $\alpha=(1+\sqrt{5}) / 2$. It is also clear that the ratio has so many applications in, specially, physics, engineering, architecture, and so forth $[5,6]$. In a similar manner, the ratio of two consecutive Padovan and Perrin numbers converges to

$$
\alpha_{P}=\sqrt[3]{\frac{1}{2}+\frac{1}{6} \sqrt{\frac{23}{3}}}+\sqrt[3]{\frac{1}{2}-\frac{1}{6} \sqrt{\frac{23}{3}}}
$$

that is named as plastic constant and was firstly defined in 1924 by Gérard Cordonnier. He described applications to architecture and illustrated the use of the plastic constant in many buildings.

Although the study of Perrin numbers started in the beginning of the 19th. century under different names, the master study was published in 2006 by Shannon et al. in [3].
The authors defined the Perrin $\left\{R_{n}\right\}_{n \in \mathbb{N}}$ and Padovan $\left\{P_{n}\right\}_{n \in \mathbb{N}}$ sequences as in the forms

$$
\begin{gathered}
R_{n+3}=R_{n+1}+R_{n}, \quad \text { where } R_{0}=3, R_{1}=0, R_{2}=2, \\
P_{n+3}=P_{n+1}+P_{n}, \quad \text { where } P_{0}=P_{1}=P_{2}=1,
\end{gathered}
$$

respectively.

On the other hand, the matrix sequences have taken so much interest for different types of numbers (cf. [7-9]). For instance, in [7], authors defined new matrix generalizations for Fibonacci and Lucas numbers, and by using basic matrix approach they showed some properties of these matrix sequences. In [9], authors defined a new sequence which generalizes $(s, t)$-Fibonacci and $(s, t)$-Lucas sequences at the same time. After that, by using it, they established generalized $(s, t)$-matrix sequence. Finally, they presented some important relationships among this new generalization, $(s, t)$-Fibonacci and $(s, t)$-Lucas sequences and their matrix sequences. In [8], Gulec and Taskara gave new generalizations for $(s, t)$-Pell and $(s, t)$-Pell Lucas sequences for Pell and PellLucas numbers. Considering these sequences, they defined the matrix sequences which have elements of $(s, t)$-Pell and $(s, t)$-Pell Lucas sequences. Also, they investigated their properties. Moreover, in [10], authors develop the matrix sequences that represent Padovan and Perrin numbers and examined their properties. 
In addition, some matrix based transforms can be introduced for a given sequence. Binomial transform is one of these transforms, and there are also other ones such as rising and falling binomial transforms (see [11-13]).

Motivated by $[10,12]$, the goal of this paper is to apply the binomial transforms to the Padovan $\left(\mathscr{P}_{n}\right)$ and Perrin matrix sequences $\left(\mathscr{R}_{n}\right)$. Also, the generating functions of these transforms are found by recurrence relations. Finally, the relations between these transforms are illustrated by deriving new formulas.

Now, we give some preliminaries related to our study. Given an integer sequence $X=\left\{x_{0}, x_{1}, x_{2}, \ldots\right\}$, the binomial transform $B$ of the sequence $X, B(X)=\left\{b_{n}\right\}$, is given by

$$
b_{n}=\sum_{i=0}^{n}\left(\begin{array}{c}
n \\
i
\end{array}\right) x_{i} .
$$

In [10], for $n \geq 0$, , authors defined Padovan and Perrin matrix sequences as in the form

$$
\mathscr{P}_{n+3}=\mathscr{P}_{n+1}+\mathscr{P}_{n},
$$

where

$$
\begin{gathered}
\mathscr{P}_{0}=\left(\begin{array}{lll}
1 & 0 & 0 \\
0 & 1 & 0 \\
0 & 0 & 1
\end{array}\right), \\
\mathscr{P}_{1}=\left(\begin{array}{lll}
0 & 1 & 0 \\
0 & 0 & 1 \\
1 & 1 & 0
\end{array}\right), \\
\mathscr{P}_{2}=\left(\begin{array}{lll}
0 & 0 & 1 \\
1 & 1 & 0 \\
0 & 1 & 1
\end{array}\right), \\
\mathscr{R}_{n+3}=\mathscr{R}_{n+1}+\mathscr{R}_{n},
\end{gathered}
$$

where

$$
\begin{aligned}
& \mathscr{R}_{0}=\left(\begin{array}{ccc}
4 & 2 & -3 \\
-3 & 1 & 2 \\
2 & -1 & 1
\end{array}\right), \\
& \mathscr{R}_{1}=\left(\begin{array}{ccc}
-3 & 1 & 2 \\
2 & -1 & 1 \\
1 & 3 & -1
\end{array}\right), \\
& \mathscr{R}_{2}=\left(\begin{array}{ccc}
2 & -1 & 1 \\
1 & 3 & -1 \\
-1 & 0 & 3
\end{array}\right) .
\end{aligned}
$$

Proposition 1 (see [10]). Let one considers $n \geq 0$, the following properties are held:

(i)

$$
\begin{aligned}
\mathscr{P}_{n} & =\left(\begin{array}{lll}
P_{n-5} & P_{n-3} & P_{n-4} \\
P_{n-4} & P_{n-2} & P_{n-3} \\
P_{n-3} & P_{n-1} & P_{n-2}
\end{array}\right), \\
\mathscr{R}_{n} & =\left(\begin{array}{lll}
R_{n-5} & R_{n-3} & R_{n-4} \\
R_{n-4} & R_{n-2} & R_{n-3} \\
R_{n-3} & R_{n-1} & R_{n-2}
\end{array}\right),
\end{aligned}
$$

(ii) for $m>j \geq 0$, the following statements are satisfied:

$$
\begin{aligned}
\sum_{i=0}^{n-1} \mathscr{P}_{m i+j} & \\
= & \left(\mathscr{P}_{m n+m+j}+\mathscr{P}_{m n-m+j}+\left(1-R_{m}\right)\right. \\
& \times \mathscr{P}_{m n+j}-\mathscr{P}_{m+j} \\
& \left.-\mathscr{P}_{m-j}+\left(R_{m}-1\right) \mathscr{P}_{j}\right) \\
& \times\left(R_{m}-R_{-m}\right)^{-1} \\
\sum_{i=0}^{n-1} \mathscr{R}_{m i+j} & \\
= & \left(\mathscr{R}_{m n+m+j}+\mathscr{R}_{m n-m+j}\right. \\
& +\left(1-R_{m}\right) \mathscr{R}_{m n+j}-\mathscr{R}_{m+j} \\
& \left.-\mathscr{R}_{m-j}+\left(R_{m}-1\right) \mathscr{R}_{j}\right) \\
& \times\left(R_{m}-R_{-m}\right)^{-1},
\end{aligned}
$$

(iii) for $m, n \geq 0$,
(a) $\mathscr{P}_{m} \mathscr{P}_{n}=\mathscr{P}_{n+m}$,
(b) $\mathscr{P}_{m} \mathscr{R}_{n}=\mathscr{R}_{n} \mathscr{P}_{m}=\mathscr{R}_{n+m}$,
(c) $\mathscr{R}_{m} \mathscr{R}_{n}=2 \mathscr{R}_{m+n-2}+\mathscr{R}_{m+n-5}$, where $m>4$ or $n>4$,
(d) $\mathscr{R}_{m} \mathscr{R}_{n}=4 \mathscr{P}_{n+m-4}+4 \mathscr{P}_{m+n-7}+\mathscr{P}_{m+n-10}$, for $m, n>4$.

\section{Binomial Transform of Padovan and Perrin Matrix Sequences}

In this section, we will mainly focus on binomial transforms of Padovan and Perrin matrix sequences to get some important results. In fact, as a middle step, we will also present the recurrence relations, Binet formulas, summations, and generating functions.

Definition 2. Let $\mathscr{P}_{n}$ and $\mathscr{R}_{n}$ be the Padovan and Perrin matrix sequences, respectively. The binomial transforms of these matrix sequences can be expressed as follows:

(i) the binomial transform of the Padovan matrix sequence is $b_{n}=\sum_{i=0}^{n}\left(\begin{array}{c}n \\ i\end{array}\right) \mathscr{P}_{i}$,

(ii) the binomial transform of the Perrin matrix sequence is $c_{n}=\sum_{i=0}^{n}\left(\begin{array}{c}n \\ i\end{array}\right) \mathscr{R}_{i}$. 
We note that, from Definition 2 and (4) and (5), for $n \geq 0$, we obtain

$$
\begin{aligned}
& b_{0}=\mathscr{P}_{0}, \\
& b_{1}=\mathscr{P}_{0}+\mathscr{P}_{1}=\mathscr{P}_{3}, \\
& b_{2}=\mathscr{P}_{0}+2 \mathscr{P}_{1}+\mathscr{P}_{2}=\mathscr{P}_{6}, \ldots, \\
& b_{n}=\mathscr{P}_{3 n}, \\
& c_{0}=\mathscr{R}_{0}, \\
& c_{1}=\mathscr{R}_{0}+\mathscr{R}_{1}=\mathscr{R}_{3}, \\
& c_{2}=\mathscr{R}_{0}+2 \mathscr{R}_{1}+\mathscr{R}_{2}=\mathscr{R}_{6}, \ldots, \\
& c_{n}=\mathscr{R}_{3 n} .
\end{aligned}
$$

The following lemma will be the key of the proof of the next theorems.

Lemma 3. For $n \geq 0$, the following equalities are held:

(i) $b_{n+1}=\sum_{i=0}^{n}\left(\begin{array}{c}n \\ i\end{array}\right)\left(\mathscr{P}_{i}+\mathscr{P}_{i+1}\right)$,

(ii) $c_{n+1}=\sum_{i=0}^{n}\left(\begin{array}{c}n \\ i\end{array}\right)\left(\mathscr{R}_{i}+\mathscr{R}_{i+1}\right)$.

Proof. Firstly, in here we will just prove (i), since (ii) can be thought in the same manner with (i).

(i) By using Definition 2 and the well known binomial equality

$$
\left(\begin{array}{c}
n+1 \\
i
\end{array}\right)=\left(\begin{array}{c}
n \\
i
\end{array}\right)+\left(\begin{array}{c}
n \\
i-1
\end{array}\right)
$$

we obtain

$$
\begin{aligned}
b_{n+1} & =\sum_{i=1}^{n+1}\left(\begin{array}{c}
n+1 \\
i
\end{array}\right) \mathscr{P}_{i}+\mathscr{P}_{0} \\
& =\sum_{i=1}^{n+1}\left(\begin{array}{c}
n \\
i
\end{array}\right) \mathscr{P}_{i}+\sum_{i=1}^{n+1}\left(\begin{array}{c}
n \\
i-1
\end{array}\right) \mathscr{P}_{i}+\mathscr{P}_{0} \\
& =\sum_{i=0}^{n}\left(\begin{array}{c}
n \\
i
\end{array}\right) \mathscr{P}_{i}+\sum_{i=0}^{n}\left(\begin{array}{c}
n \\
i
\end{array}\right) \mathscr{P}_{i+1} \\
& =\sum_{i=0}^{n}\left(\begin{array}{c}
n \\
i
\end{array}\right)\left(\mathscr{P}_{i}+\mathscr{P}_{i+1}\right),
\end{aligned}
$$

which is a desired result.

From the previous Lemma, note that

(i) $b_{n+1}$ also can be written as $b_{n+1}=b_{n}+\sum_{i=0}^{n}\left(\begin{array}{c}n \\ i\end{array}\right) \mathscr{P}_{i+1}$,

(ii) $c_{n+1}$ also can be written as $c_{n+1}=c_{n}+\sum_{i=0}^{n}\left(\begin{array}{c}n \\ i\end{array}\right) \mathscr{R}_{i+1}$.

Theorem 4. For $n>0$,

(i) recurrence relation of sequences $\left\{b_{n}\right\}$ is

$$
b_{n+2}=3 b_{n+1}-2 b_{n}+b_{n-1},
$$

with initial conditions

$$
\begin{aligned}
& b_{0}=\left(\begin{array}{lll}
1 & 0 & 0 \\
0 & 1 & 0 \\
0 & 0 & 1
\end{array}\right), \\
& b_{1}=\left(\begin{array}{lll}
1 & 1 & 0 \\
0 & 1 & 1 \\
1 & 1 & 1
\end{array}\right), \\
& b_{2}=\left(\begin{array}{lll}
1 & 2 & 1 \\
1 & 2 & 2 \\
2 & 3 & 2
\end{array}\right),
\end{aligned}
$$

(ii) recurrence relation of sequences $\left\{c_{n}\right\}$ is

$$
c_{n+2}=3 c_{n+1}-2 c_{n}+c_{n-1} \text {, }
$$

with initial conditions

$$
\begin{gathered}
c_{0}=\left(\begin{array}{ccc}
4 & 2 & -3 \\
-3 & 1 & 2 \\
2 & -1 & 1
\end{array}\right), \\
c_{1}=\left(\begin{array}{ccc}
1 & 3 & -1 \\
-1 & 0 & 3 \\
3 & 2 & 0
\end{array}\right), \\
c_{2}=\left(\begin{array}{lll}
0 & 3 & 2 \\
2 & 2 & 3 \\
3 & 5 & 2
\end{array}\right) .
\end{gathered}
$$

Proof. Similarly for the proof of the previous theorem, only the first case (i) will be proved. We will omit the other cases since the proofs will not be different.

(i) By considering the right-hand side of equality in (i) and Definition 2, we obtain

$$
\begin{aligned}
3 b_{n+1}- & 2 b_{n}+b_{n-1} \\
= & 3 \sum_{i=0}^{n+1}\left(\begin{array}{c}
n+1 \\
i
\end{array}\right) \mathscr{P}_{i}-2 \sum_{i=0}^{n}\left(\begin{array}{c}
n \\
i
\end{array}\right) \mathscr{P}_{i}+\sum_{i=0}^{n-1}\left(\begin{array}{c}
n-1 \\
i
\end{array}\right) \mathscr{P}_{i} \\
= & \sum_{i=0}^{n+1}\left(\begin{array}{c}
n+1 \\
i
\end{array}\right) \mathscr{P}_{i}+2 \sum_{i=0}^{n+1}\left[\left(\begin{array}{c}
n+1 \\
i
\end{array}\right)-\left(\begin{array}{c}
n \\
i
\end{array}\right)\right] \mathscr{P}_{i} \\
& +\sum_{i=0}^{n-1}\left(\begin{array}{c}
n-1 \\
i
\end{array}\right) \mathscr{P}_{i} \\
= & \sum_{i=0}^{n+1}\left(\begin{array}{c}
n+1 \\
i
\end{array}\right) \mathscr{P}_{i}+2 \sum_{i=0}^{n+1}\left(\begin{array}{c}
n \\
i-1
\end{array}\right) \mathscr{P}_{i} \\
& +\sum_{i=0}^{n-1}\left(\begin{array}{c}
n-1 \\
i
\end{array}\right) \mathscr{P}_{i} .
\end{aligned}
$$


By taking, account equality $\left(\begin{array}{c}n+1 \\ n\end{array}\right)=\left(\begin{array}{c}n \\ -1\end{array}\right)=0$, we get

$$
\begin{aligned}
& 3 b_{n+1}-2 b_{n}+b_{n-1} \\
& =\sum_{i=0}^{n+1}\left(\begin{array}{c}
n+1 \\
i
\end{array}\right) \mathscr{P}_{i}+2 \sum_{i=1}^{n+1}\left(\begin{array}{c}
n \\
i-1
\end{array}\right) \mathscr{P}_{i} \\
& +\sum_{i=0}^{n-1}\left(\begin{array}{c}
n-1 \\
i
\end{array}\right) \mathscr{P}_{i} \\
& =\sum_{i=0}^{n+1}\left(\begin{array}{c}
n+1 \\
i
\end{array}\right) \mathscr{P}_{i}+\sum_{i=0}^{n}\left(\begin{array}{c}
n \\
i
\end{array}\right) \mathscr{P}_{i+1}+\sum_{i=1}^{n+1}\left(\begin{array}{c}
n \\
i-1
\end{array}\right) \mathscr{P}_{i} \\
& +\sum_{i=0}^{n-1}\left(\begin{array}{c}
n-1 \\
i
\end{array}\right) \mathscr{P}_{i} \\
& =\sum_{i=0}^{n+1}\left(\begin{array}{c}
n+1 \\
i
\end{array}\right) \mathscr{P}_{i}+\sum_{i=0}^{n+1}\left(\begin{array}{c}
n \\
i
\end{array}\right) \mathscr{P}_{i+1}+\sum_{i=1}^{n+1}\left(\begin{array}{c}
n \\
i-1
\end{array}\right) \mathscr{P}_{i} \\
& +\sum_{i=0}^{n-1}\left(\begin{array}{c}
n-1 \\
i
\end{array}\right) \mathscr{P}_{i}+\sum_{i=0}^{n+1}\left(\begin{array}{c}
n \\
i-1
\end{array}\right) \mathscr{P}_{i+1} \\
& -\sum_{i=0}^{n+1}\left(\begin{array}{c}
n \\
i-1
\end{array}\right) \mathscr{P}_{i+1} \\
& =\sum_{i=0}^{n+1}\left(\begin{array}{c}
n+1 \\
i
\end{array}\right) \mathscr{P}_{i}+\sum_{i=0}^{n+1}\left[\left(\begin{array}{c}
n \\
i
\end{array}\right)+\left(\begin{array}{c}
n \\
i-1
\end{array}\right)\right] \\
& \times \mathscr{P}_{i+1}+\sum_{i=1}^{n+1}\left(\begin{array}{c}
n \\
i-1
\end{array}\right) \mathscr{P}_{i} \\
& +\sum_{i=0}^{n-1}\left(\begin{array}{c}
n-1 \\
i
\end{array}\right) \mathscr{P}_{i}-\sum_{i=1}^{n+1}\left(\begin{array}{c}
n \\
i-1
\end{array}\right) \mathscr{P}_{i+1} \\
& =\sum_{i=0}^{n+1}\left(\begin{array}{c}
n+1 \\
i
\end{array}\right) \mathscr{P}_{i}+\sum_{i=0}^{n+1}\left(\begin{array}{c}
n+1 \\
i
\end{array}\right) \mathscr{P}_{i+1} \\
& +\sum_{i=1}^{n+1}\left(\begin{array}{c}
n \\
i-1
\end{array}\right) \mathscr{P}_{i} \\
& +\sum_{i=0}^{n-1}\left(\begin{array}{c}
n-1 \\
i
\end{array}\right) \mathscr{P}_{i}-\sum_{i=1}^{n+1}\left(\begin{array}{c}
n \\
i-1
\end{array}\right) \mathscr{P}_{i+1} .
\end{aligned}
$$

From Lemma 3 and properties of binomial sum, we have

$$
\begin{aligned}
3 b_{n+1} & -2 b_{n}+b_{n-1} \\
& =b_{n+2}+\sum_{i=0}^{n}\left(\begin{array}{c}
n \\
i
\end{array}\right) \mathscr{P}_{i+1}+\sum_{i=0}^{n-1}\left(\begin{array}{c}
n-1 \\
i
\end{array}\right) \mathscr{P}_{i}-\sum_{i=0}^{n}\left(\begin{array}{c}
n \\
i
\end{array}\right) \mathscr{P}_{i+2} .
\end{aligned}
$$

On the other hand, by using (4) and the equality $\left(\begin{array}{c}n \\ -1\end{array}\right)=0$, we get

$$
\begin{aligned}
3 b_{n+1}= & 2 b_{n}+b_{n-1} \\
= & b_{n+2}+\sum_{i=0}^{n-1}\left(\begin{array}{c}
n-1 \\
i
\end{array}\right) \mathscr{P}_{i}-\sum_{i=0}^{n}\left(\begin{array}{c}
n \\
i
\end{array}\right) \mathscr{P}_{i-3} \\
= & b_{n+2}+\sum_{i=0}^{n-1}\left(\begin{array}{c}
n-1 \\
i
\end{array}\right) \mathscr{P}_{i} \\
& -\sum_{i=0}^{n}\left[\left(\begin{array}{c}
n-1 \\
i
\end{array}\right)+\left(\begin{array}{c}
n-1 \\
i-1
\end{array}\right)\right] \mathscr{P}_{i-3} \\
= & b_{n+2}+\sum_{i=0}^{n-1}\left(\begin{array}{c}
n-1 \\
i
\end{array}\right) \mathscr{P}_{i}-\sum_{i=0}^{n}\left(\begin{array}{c}
n-1 \\
i
\end{array}\right) \mathscr{P}_{i-3} \\
& -\sum_{i=1}^{n}\left(\begin{array}{c}
n-1 \\
i-1
\end{array}\right) \mathscr{P}_{i-3} \\
= & b_{n+2}+\sum_{i=0}^{n-1}\left(\begin{array}{c}
n-1 \\
i
\end{array}\right) \mathscr{P}_{i}-\sum_{i=0}^{n}\left(\begin{array}{c}
n-1 \\
i
\end{array}\right) \mathscr{P}_{i-3} \\
& -\sum_{i=0}^{n-1}\left(\begin{array}{c}
n-1 \\
i
\end{array}\right) \mathscr{P}_{i-2} \\
= & b_{n+2}+\sum_{i=0}^{n-1}\left(\begin{array}{c}
n-1 \\
i
\end{array}\right)\left(\mathscr{P}_{i}-\mathscr{P}_{i-2}-\mathscr{P}_{i-3}\right) \\
= & b_{n+2},
\end{aligned}
$$

which has completed the proof of this case.

The characteristic equation of sequences $\left\{b_{n}\right\}$ and $\left\{c_{n}\right\}$ in (13) and (15) is $\lambda^{3}-3 \lambda^{2}+2 \lambda-1=0$. Let $\lambda_{1}, \lambda_{2}$, and $\lambda_{3}$ be the roots of this equation. Then, Binet's formulas of sequences $\left\{b_{n}\right\}$ and $\left\{c_{n}\right\}$ can be expressed as

$$
\begin{aligned}
& b_{n}=X_{1} \lambda_{1}^{n}+Y_{1} \lambda_{2}^{n}+Z_{1} \lambda_{3}^{n}, \\
& c_{n}=X_{2} \lambda_{1}^{n}+Y_{2} \lambda_{2}^{n}+Z_{2} \lambda_{3}^{n},
\end{aligned}
$$

where

$$
\begin{aligned}
X_{1}= & \frac{1}{\lambda_{1}\left(\lambda_{1}-\lambda_{2}\right)\left(\lambda_{1}-\lambda_{3}\right)} \\
& \times\left(\begin{array}{ccc}
\lambda_{1}^{2}-2 \lambda_{1}+1 & \lambda_{1}^{2}-\lambda_{1} & \lambda_{1} \\
\lambda_{1} & \lambda_{1}^{2}-\lambda_{1}+1 & \lambda_{1}^{2}-\lambda_{1} \\
\lambda_{1}^{2}-\lambda_{1} & \lambda_{1}^{2} & \lambda_{1}^{2}-\lambda_{1}+1
\end{array}\right), \\
Y_{1}= & \frac{1}{\lambda_{2}\left(\lambda_{2}-\lambda_{1}\right)\left(\lambda_{2}-\lambda_{3}\right)} \\
& \times\left(\begin{array}{ccc}
\lambda_{2}^{2}-2 \lambda_{2}+1 & \lambda_{2}^{2}-\lambda_{2} & \lambda_{2} \\
\lambda_{2} & \lambda_{2}^{2}-\lambda_{2}+1 & \lambda_{2}^{2}-\lambda_{2} \\
\lambda_{2}^{2}-\lambda_{2} & \lambda_{2}^{2} & \lambda_{2}^{2}-\lambda_{2}+1
\end{array}\right),
\end{aligned}
$$




$$
\begin{aligned}
& Z_{1}=\frac{1}{\lambda_{3}\left(\lambda_{3}-\lambda_{1}\right)\left(\lambda_{3}-\lambda_{2}\right)} \\
& \times\left(\begin{array}{ccc}
\lambda_{3}^{2}-2 \lambda_{3}+1 & \lambda_{3}^{2}-\lambda_{3} & \lambda_{3} \\
\lambda_{3} & \lambda_{3}^{2}-\lambda_{3}+1 & \lambda_{3}^{2}-\lambda_{3} \\
\lambda_{3}^{2}-\lambda_{3} & \lambda_{3}^{2} & \lambda_{3}^{2}-\lambda_{3}+1
\end{array}\right), \\
& X_{2}=\frac{1}{\lambda_{1}\left(\lambda_{1}-\lambda_{2}\right)\left(\lambda_{1}-\lambda_{3}\right)} \\
& \times\left(\begin{array}{ccc}
\lambda_{1}^{2}-3 \lambda_{1}+4 & 3 \lambda_{1}^{2}-6 \lambda_{1}+2 & -\lambda_{1}^{2}+5 \lambda_{1}-3 \\
-\lambda_{1}^{2}+5 \lambda_{1}-3 & 2 \lambda_{1}+1 & 3 \lambda_{1}^{2}-6 \lambda_{1}+2 \\
3 \lambda_{1}^{2}-6 \lambda_{1}+2 & 2 \lambda_{1}^{2}-\lambda_{1}-1 & 2 \lambda_{1}+1
\end{array}\right), \\
& Y_{2}=\frac{1}{\lambda_{2}\left(\lambda_{2}-\lambda_{1}\right)\left(\lambda_{2}-\lambda_{3}\right)} \\
& \times\left(\begin{array}{ccc}
\lambda_{2}^{2}-3 \lambda_{2}+4 & 3 \lambda_{2}^{2}-6 \lambda_{2}+2 & -\lambda_{2}^{2}+5 \lambda_{2}-3 \\
-\lambda_{2}^{2}+5 \lambda_{2}-3 & 2 \lambda_{2}+1 & 3 \lambda_{2}^{2}-6 \lambda_{2}+2 \\
3 \lambda_{2}^{2}-6 \lambda_{2}+2 & 2 \lambda_{2}^{2}-\lambda_{2}-1 & 2 \lambda_{2}+1
\end{array}\right), \\
& Z_{2}=\frac{1}{\lambda_{3}\left(\lambda_{3}-\lambda_{1}\right)\left(\lambda_{3}-\lambda_{2}\right)} \\
& \times\left(\begin{array}{ccc}
\lambda_{3}^{2}-3 \lambda_{3}+4 & 3 \lambda_{3}^{2}-6 \lambda_{3}+2 & -\lambda_{3}^{2}+5 \lambda_{3}-3 \\
-\lambda_{3}^{2}+5 \lambda_{3}-3 & 2 \lambda_{3}+1 & 3 \lambda_{3}^{2}-6 \lambda_{3}+2 \\
3 \lambda_{3}^{2}-6 \lambda_{3}+2 & 2 \lambda_{3}^{2}-\lambda_{3}-1 & 2 \lambda_{3}+1
\end{array}\right) .
\end{aligned}
$$

Now, we give the sums of binomial transforms for Padovan and Perrin matrix sequences.

Theorem 5. Sums of sequences $\left\{b_{n}\right\}$ and $\left\{c_{n}\right\}$ are

(i) $\sum_{k=0}^{n-1} b_{k}=\mathscr{P}_{3 n-1}-2 \mathscr{P}_{1}$,

(ii) $\sum_{k=0}^{n-1} c_{k}=\mathscr{R}_{3 n-1}-2 \mathscr{R}_{1}$.

Proof. (i) By considering (9), we have

$$
\sum_{k=0}^{n-1} b_{k}=\sum_{k=0}^{n-1} \mathscr{P}_{3 k}
$$

Now, if we take $m=3$, and $j=0$ in first equality of Proposition 1-(ii), then we obtain

$$
\begin{aligned}
\sum_{k=0}^{n-1} b_{k}=\left(\mathscr{P}_{3 n+3}+\mathscr{P}_{3 n-3}+(1-3) \mathscr{P}_{3 n}\right. \\
\left.\quad-2 \mathscr{P}_{3}+(3-1) \mathscr{P}_{0}\right) \times(3-2)^{-1} .
\end{aligned}
$$

Afterwards, by taking into account (4), we conclude

$$
\sum_{k=0}^{n-1} b_{k}=\mathscr{P}_{3 n-1}-2 \mathscr{P}_{1} .
$$

(ii) The proof of the binomial transform of Perrin matrix sequences can be seen by taking into account (10), Proposition 1-(ii) and (5), similarly to the proof of (i).

Theorem 6. The generating functions of the binomial transforms for $\left\{\mathscr{P}_{n}\right\}$ and $\left\{\mathscr{R}_{n}\right\}$ are

(i)

$$
\begin{aligned}
\sum_{i=0}^{\infty} b_{i} x^{i}= & \frac{1}{1-3 x+2 x^{2}-x^{3}} \\
& \times\left(\begin{array}{ccc}
1-2 x & x-x^{2} & x^{2} \\
x^{2} & 1-2 x+x^{2} & x-x^{2} \\
x-x^{2} & x & 1-2 x+x^{2}
\end{array}\right),
\end{aligned}
$$

(ii)

$$
\begin{aligned}
\sum_{i=0}^{\infty} c_{i} x^{i}= & \frac{1}{1-3 x+2 x^{2}-x^{3}} \\
& \times\left(\begin{array}{ccc}
4-11 x+5 x^{2} & 2-3 x-2 x^{2} & -3+8 x-x^{2} \\
-3+8 x-x^{2} & 1-3 x+4 x^{2} & 2-3 x-2 x^{2} \\
2-3 x-2 x^{2} & -1+5 x-3 x^{2} & 1-3 x+4 x^{2}
\end{array}\right)
\end{aligned}
$$

respectively.

Proof. We omit Padovan case since the proof will be quite similar.

Assume that $c(x)$ is the generating function of the binomial transform for $\left\{\mathscr{R}_{n}\right\}$. Then, we have

$$
c(x)=\sum_{i=0}^{\infty} c_{i} x^{i}
$$

From Theorem 4, we obtain

$$
\begin{aligned}
c(x)= & c_{0}+c_{1} x+c_{2} x^{2}+\sum_{i=3}^{\infty}\left(3 c_{i-1}-2 c_{i-2}+c_{i-3}\right) x^{i} \\
= & c_{0}+c_{1} x+c_{2} x^{2}-3 c_{0} x-3 c_{1} x^{2}+2 c_{0} x^{2}+3 x \sum_{i=0}^{\infty} c_{i} x^{i} \\
& -2 x^{2} \sum_{i=0}^{\infty} c_{i} x^{i}+x^{3} \sum_{i=0}^{\infty} c_{i} x^{i} \\
= & c_{0}+\left(c_{1}-3 c_{0}\right) x+\left(c_{2}-3 c_{1}+2 c_{0}\right) x^{2} \\
& +3 x c(x)-2 x^{2} c(x)+x^{3} c(x) .
\end{aligned}
$$

Now, the rearrangement of the equation implies that

$$
c(x)=\frac{c_{0}+\left(c_{1}-3 c_{0}\right) x+\left(c_{2}-3 c_{1}+2 c_{0}\right) x^{2}}{1-3 x+2 x^{2}-x^{3}},
$$

which is equal to the $\sum_{i=0}^{\infty} c_{i} x^{i}$ in theorem.

Hence, the result is obtained. 


\section{The Relationships between New Binomial Transforms}

In this section, we present the relationship between these binomial transforms.

Theorem 7. For $n, m \geq 0$, one has

(i) $b_{n} b_{m}=b_{n+m}$, where $n \leq m$,

(ii) $b_{n} c_{m}=c_{n} b_{m}=c_{n+m}$,

(iii) $c_{n} c_{m}=2 c_{n+m}-c_{n+m-1}-c_{n+m-2}$, where $m>1$ or $n>1$,

(iv) $c_{n} c_{m}=8 b_{n+m}-15 b_{n+m-1}+2 b_{n+m-2}$, where $m, n>1$.

Proof. (i) From Definition 2, we have

$$
\begin{aligned}
b_{n} b_{m}= & \left(\sum_{i=0}^{n}\left(\begin{array}{c}
n \\
i
\end{array}\right) \mathscr{P}_{i}\right)\left(\sum_{j=0}^{m}\left(\begin{array}{c}
m \\
j
\end{array}\right) \mathscr{P}_{j}\right) \\
= & {\left[\left(\begin{array}{c}
n \\
0
\end{array}\right) \mathscr{P}_{0}+\left(\begin{array}{c}
n \\
1
\end{array}\right) \mathscr{P}_{1}+\cdots+\left(\begin{array}{c}
n \\
n
\end{array}\right) \mathscr{P}_{n}\right] } \\
& \times\left[\left(\begin{array}{c}
m \\
0
\end{array}\right) \mathscr{P}_{0}+\left(\begin{array}{c}
m \\
1
\end{array}\right) \mathscr{P}_{1}+\cdots+\left(\begin{array}{c}
m \\
m
\end{array}\right) \mathscr{P}_{m}\right] .
\end{aligned}
$$

By considering Proposition 1-(iii), we obtain

$$
\begin{aligned}
& b_{n} b_{m}=\left(\begin{array}{l}
n \\
0
\end{array}\right)\left(\begin{array}{c}
m \\
0
\end{array}\right) \mathscr{P}_{0}+\left(\begin{array}{c}
n \\
0
\end{array}\right)\left(\begin{array}{c}
m \\
1
\end{array}\right) \mathscr{P}_{1}+\cdots+\left(\begin{array}{l}
n \\
0
\end{array}\right)\left(\begin{array}{l}
m \\
m
\end{array}\right) \mathscr{P}_{m} \\
&+\left(\begin{array}{c}
n \\
1
\end{array}\right)\left(\begin{array}{c}
m \\
0
\end{array}\right) \mathscr{P}_{1}+\left(\begin{array}{c}
n \\
1
\end{array}\right)\left(\begin{array}{c}
m \\
1
\end{array}\right) \mathscr{P}_{2}+\cdots \\
&+\left(\begin{array}{c}
n \\
1
\end{array}\right)\left(\begin{array}{c}
m \\
m
\end{array}\right) \mathscr{P}_{m+1}+ \\
& \vdots \\
&+\left(\begin{array}{c}
n \\
n
\end{array}\right)\left(\begin{array}{c}
m \\
0
\end{array}\right) \mathscr{P}_{n}+\left(\begin{array}{c}
n \\
n
\end{array}\right)\left(\begin{array}{c}
m \\
1
\end{array}\right) \mathscr{P}_{n+1}+\cdots \\
&+\left(\begin{array}{c}
n \\
n
\end{array}\right)\left(\begin{array}{c}
m \\
m
\end{array}\right) \mathscr{P}_{n+m} \\
&=\left(\begin{array}{c}
n \\
0
\end{array}\right)\left(\begin{array}{c}
m \\
0
\end{array}\right) \mathscr{P}_{0}+\left[\left(\begin{array}{c}
n \\
0
\end{array}\right)\left(\begin{array}{c}
m \\
1
\end{array}\right)+\left(\begin{array}{c}
n \\
1
\end{array}\right)\left(\begin{array}{c}
m \\
0
\end{array}\right)\right] \mathscr{P}_{1} \\
&+\left(\left(\begin{array}{c}
n \\
0
\end{array}\right)\left(\begin{array}{c}
m \\
2
\end{array}\right)+\left(\begin{array}{c}
n \\
1
\end{array}\right)\left(\begin{array}{c}
m \\
1
\end{array}\right)+\left(\begin{array}{c}
n \\
2
\end{array}\right)\left(\begin{array}{c}
m \\
0
\end{array}\right)\right] \mathscr{P}_{2}+\cdots \\
&+\left[\left(\begin{array}{c}
n \\
0
\end{array}\right)\left(\begin{array}{c}
m \\
k
\end{array}\right)+\left(\begin{array}{c}
n \\
1
\end{array}\right)\left(\begin{array}{c}
m \\
k-1
\end{array}\right)\right. \\
&\left.+\cdots+\left(\begin{array}{l}
n \\
k
\end{array}\right)\left(\begin{array}{c}
m \\
0
\end{array}\right)\right] \mathscr{P}_{k}+\cdots \\
&
\end{aligned}
$$

By taking into account Vandermonde's identity $\sum_{j=0}^{k}\left(\begin{array}{l}x \\ j\end{array}\right)\left(\begin{array}{c}y \\ k-j\end{array}\right)=\left(\begin{array}{c}x+y \\ k\end{array}\right)$, we get

$$
\begin{aligned}
b_{n} b_{m}= & \left(\begin{array}{c}
n+m \\
0
\end{array}\right) \mathscr{P}_{0}+\left(\begin{array}{c}
n+m \\
1
\end{array}\right) \mathscr{P}_{1}+\left(\begin{array}{c}
n+m \\
2
\end{array}\right) \mathscr{P}_{2}+\cdots \\
& +\left(\begin{array}{c}
n+m \\
k
\end{array}\right) \mathscr{P}_{k}+\cdots+\left(\begin{array}{c}
n+m \\
n+m
\end{array}\right) \mathscr{P}_{n+m} \\
= & \sum_{i=0}^{n+m}\left(\begin{array}{c}
n+m \\
i
\end{array}\right) \mathscr{P}_{i} \\
= & b_{n+m} .
\end{aligned}
$$

(ii) Here, we will just show that the truthness of the equality $b_{n} c_{m}=c_{n+m}$, since the other can be done similarly. By considering (9), (10), and Proposition 1-(iii), we obtain

$$
b_{n} c_{m}=\mathscr{P}_{3 n} \mathscr{R}_{3 m}=\mathscr{R}_{3 n+3 m}=c_{n+m} .
$$

(iii) By considering (10) and Proposition 1-(iii), we obtain

$$
c_{n} c_{m}=\mathscr{R}_{3 n} \mathscr{R}_{3 m}=2 \mathscr{R}_{3 n+3 m-2}+\mathscr{R}_{3 n+3 m-5} .
$$

From (5), we have

$$
\begin{aligned}
c_{n} c_{m}= & 2\left(\mathscr{R}_{3 n+3 m}-\mathscr{R}_{3 n+3 m-3}\right) \\
& +\mathscr{R}_{3 n+3 m-3}-\mathscr{R}_{3 n+3 m-6} \\
= & 2 \mathscr{R}_{3 n+3 m}-\mathscr{R}_{3 n+3 m-3}-\mathscr{R}_{3 n+3 m-6} .
\end{aligned}
$$

Now, by taking into account again (10), we get $c_{n} c_{m}=$ $2 c_{n+m}-c_{n+m-1}-c_{n+m-2}$, as required.

The final part of the proof can be seen similarly as in the proof of (iii).

Theorem 8. The properties of the transforms $\left\{b_{n}\right\}$ and $\left\{c_{n}\right\}$ would be illustrated by following way:

$$
\begin{aligned}
\text { (i) } b_{n+1}-b_{n} & =\mathscr{P}_{1} b_{n} \text {, } \\
\text { (ii) } c_{n+1}-c_{n} & =\mathscr{P}_{1} c_{n} \text {, } \\
\text { (iii) } c_{n+1}-c_{n} & =\mathscr{R}_{1} b_{n} .
\end{aligned}
$$

Proof. We will omit the proof of (ii) and (iii), since it is quite similar to (i). Therefore, by considering Definition 2 and Lemma 3-(i), we have

$$
\begin{aligned}
b_{n+1}-b_{n} & =\sum_{i=0}^{n}\left(\begin{array}{c}
n \\
i
\end{array}\right)\left(\mathscr{P}_{i+1}+\mathscr{P}_{i}\right)-\sum_{i=0}^{n}\left(\begin{array}{c}
n \\
i
\end{array}\right) \mathscr{P}_{i} \\
& =\sum_{i=0}^{n}\left(\begin{array}{c}
n \\
i
\end{array}\right) \mathscr{P}_{i+1} .
\end{aligned}
$$

From Proposition 1-(iii), we get

$$
b_{n+1}-b_{n}=\sum_{i=0}^{n}\left(\begin{array}{c}
n \\
i
\end{array}\right) \mathscr{P}_{1} \mathscr{P}_{i}=\mathscr{P}_{1} b_{n} .
$$


Theorem 9. For $n, m \geq 0$, the relation between the transforms $\left\{b_{n}\right\}$ and $\left\{c_{n}\right\}$ is

$$
\mathscr{R}_{m} b_{n}=\mathscr{P}_{m} c_{n}
$$

Proof. By considering Definition 2, we have

$$
\begin{aligned}
\mathscr{R}_{m} b_{n} & =\mathscr{R}_{m} \sum_{i=0}^{n}\left(\begin{array}{c}
n \\
i
\end{array}\right) \mathscr{P}_{i} \\
& =\sum_{i=0}^{n}\left(\begin{array}{c}
n \\
i
\end{array}\right) \mathscr{R}_{m} \mathscr{P}_{i} .
\end{aligned}
$$

From Proposition 1-(iii), we get

$$
\begin{aligned}
\mathscr{R}_{m} b_{n} & =\sum_{i=0}^{n}\left(\begin{array}{c}
n \\
i
\end{array}\right) \mathscr{R}_{m+i} \\
& =\sum_{i=0}^{n}\left(\begin{array}{c}
n \\
i
\end{array}\right) \mathscr{R}_{i} \mathscr{P}_{m} \\
& =\mathscr{P}_{m} c_{n} .
\end{aligned}
$$

By choosing $m=0$ in Theorem 9 and using the initial conditions of (4) and (5), we obtain the following corollary.

Corollary 10. The following equalities are held:

(i) $c_{n}=\mathscr{R}_{0} b_{n}$,

(ii) $b_{n}=\mathscr{R}_{0}^{-1} c_{n}$.

\section{Conflict of Interests}

The authors declare that there is no conflict of interests regarding the publication of this paper.

\section{References}

[1] S. Falcón and A. Plaza, "On the Fibonacci $k$-numbers," Chaos, Solitons \& Fractals, vol. 32, no. 5, pp. 1615-1624, 2007.

[2] T. Koshy, Fibonacci and Lucas Numbers with Applications, Pure and Applied Mathematics, Wiley-Interscience, New York, NY, USA, 2001.

[3] A. G. Shannon, P. G. Anderson, and A. F. Horadam, "Properties of Cordonnier, Perrin and Van der Laan numbers," International Journal of Mathematical Education in Science and Technology, vol. 37, no. 7, pp. 825-831, 2006.

[4] Y. Yazlik and N. Taskara, "A note on generalized $k$-Horadam sequence," Computers \& Mathematics with Applications, vol. 63, no. 1, pp. 36-41, 2012.

[5] L. Marek-Crnjac, "On the mass spectrum of the elementary particles of the standard model using El Naschie's golden field theory," Chaos, Solutions \& Fractals, vol. 15, no. 4, pp. 611-618, 2003.

[6] L. Marek-Crnjac, "The mass spectrum of high energy elementary particles via El Naschie's $E^{(\infty)}$ golden mean nested oscillators, the Dunkerly-Southwell eigenvalue theorems and KAM," Chaos, Solutions \& Fractals, vol. 18, no. 1, pp. 125-133, 2003.
[7] H. Civciv and R. Türkmen, "On the $(s, t)$-Fibonacci and Fibonacci matrix sequences," Ars Combinatoria, vol. 87, pp. 161173, 2008.

[8] H. H. Gulec and N. Taskara, "On the $(s, t)$-Pell and $(s, t)$ Pell-Lucas sequences and their matrix representations," Applied Mathematics Letters, vol. 25, no. 10, pp. 1554-1559, 2012.

[9] Y. Yazlik, N. Taskara, K. Uslu, and N. Yilmaz, "The generalized $(s, t)$-sequence and its matrix sequence," AIP Conference Proceedings, vol. 1389, pp. 381-384, 2012.

[10] N. Yilmaz and N. Taskara, "Matrix sequences in terms of Padovan and Perrin numbers," Journal of Applied Mathematics. In press.

[11] K.-W. Chen, "Identities from the binomial transform," Journal of Number Theory, vol. 124, no. 1, pp. 142-150, 2007.

[12] S. Falcon and A. Plaza, "Binomial transforms of $k$-Fibonacci sequence," International Journal of Nonlinear Sciences and Numerical Simulation, vol. 10, no. 11-12, pp. 1527-1538, 2009.

[13] H. Prodinger, "Some information about the binomial transform," The Fibonacci Quarterly, vol. 32, no. 5, pp. 412-415, 1994. 


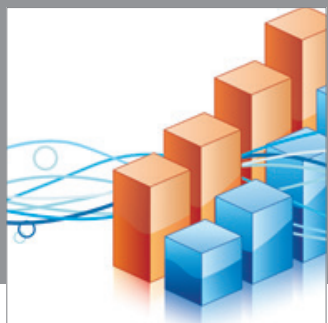

Advances in

Operations Research

mansans

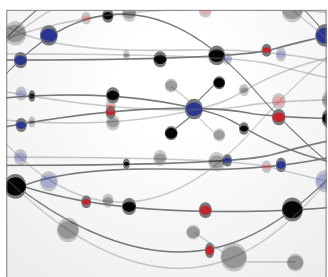

The Scientific World Journal
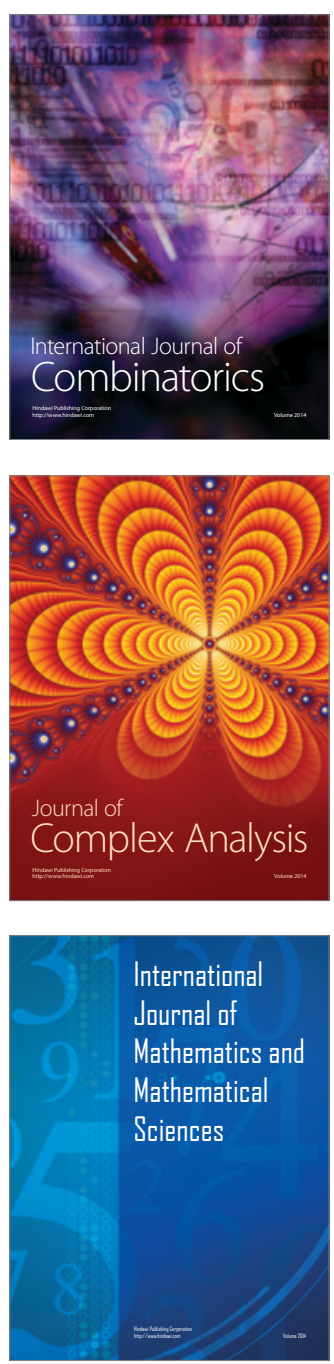
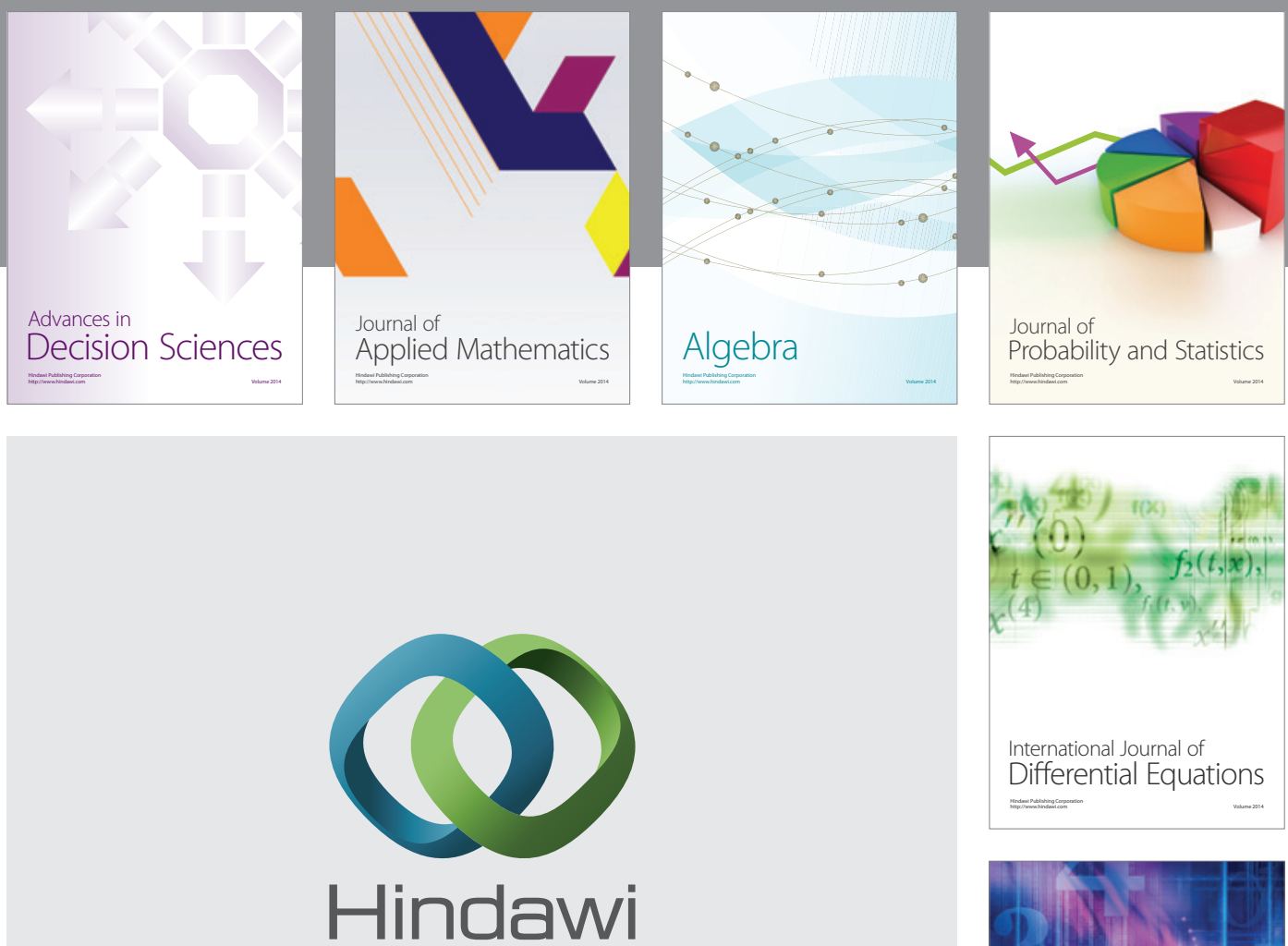

Submit your manuscripts at http://www.hindawi.com
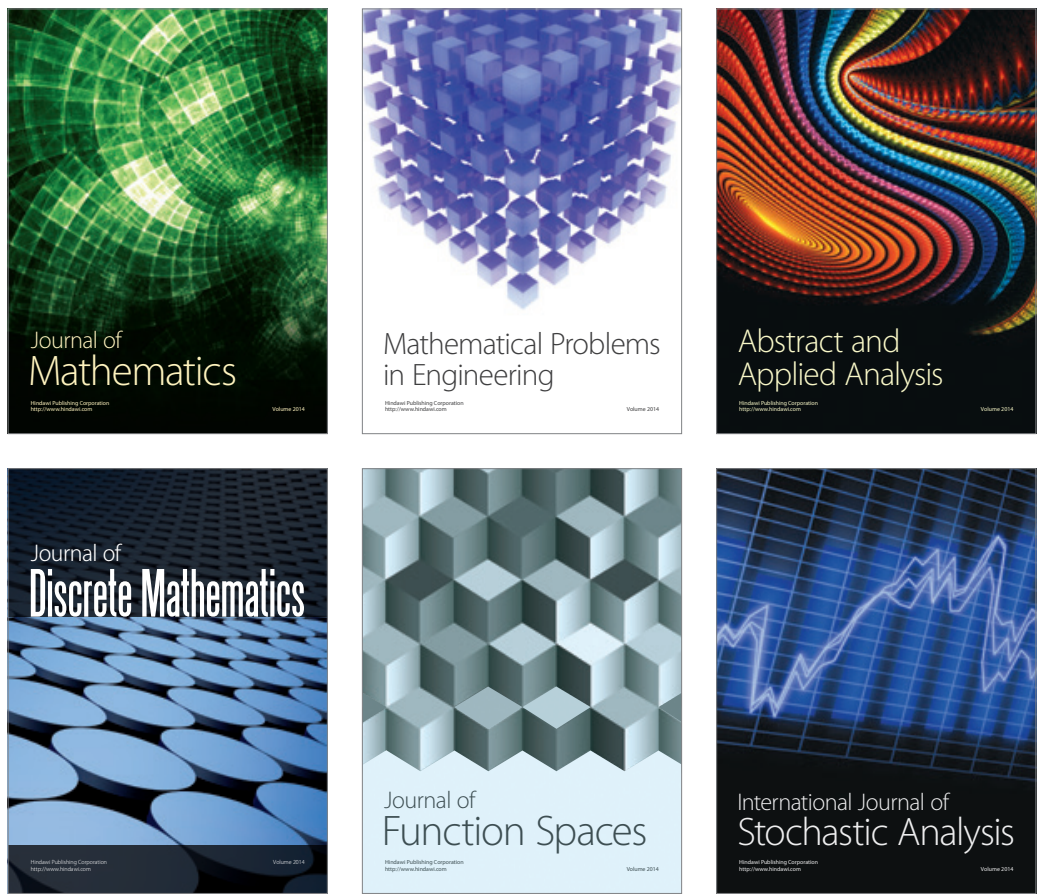

Journal of

Function Spaces

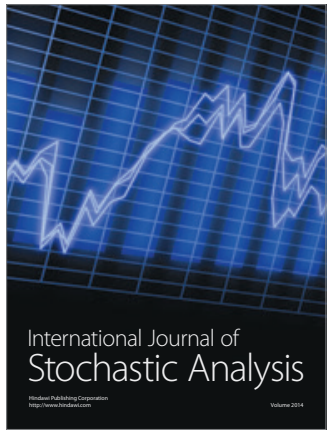

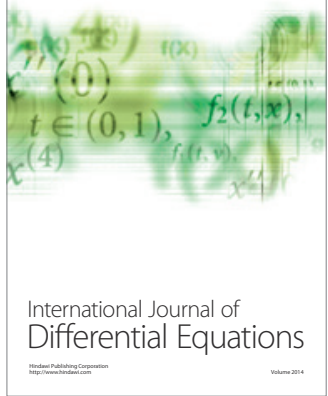
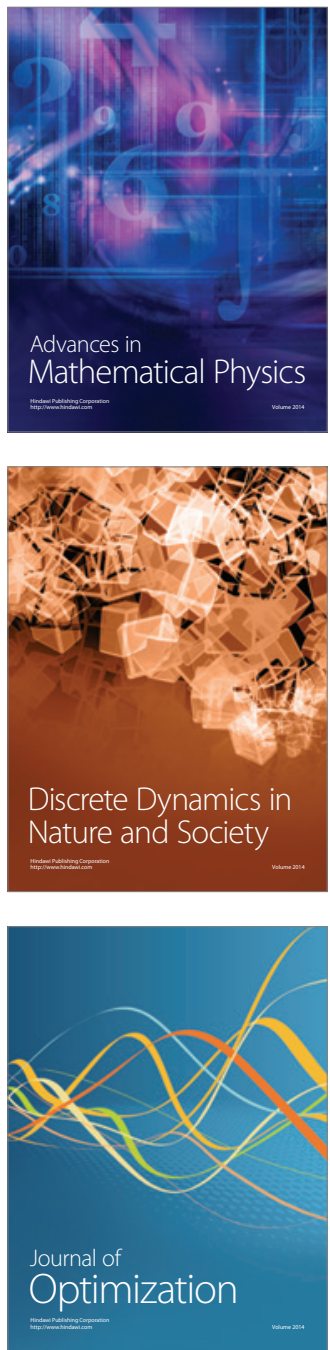